\section{Première décision embryonnaire : adopter un destin de trophectoderme ou de masse interne?}

Laure Coulombel
Directrice de recherche émérite, Inserm U1130, conseillère scientifique de médecine/sciences, Paris, France. Laure.coulombel@inserm.fr
> Comprendre comment se détermine le destin d'une cellule est l'un des défis les plus excitants de la recherche en biologie. Pouvoir l'explorer in vivo, dans le contexte physiologique, était jusqu'à il y a peu hors de portée expérimentale, a fortiori s'agissant d'embryons préimplantatoires, qui ne contiennent que 8 à 16 cellules. Les progrès de la résolution dans le temps et l'espace de l'imagerie en temps réel, la qualité des systèmes de traçage avec des protéinesétiquettes fluorescentes, l'apport de la modélisation, la miniaturisation des techniques de manipulation cellulaire ont bouleversé ce champ d'investigation, et permettent aujourd'hui non seulement de manipuler des cellules uniques, mais aussi de suivre leur cinétique de développement in vivo. Une équipe d'Heidelberg, dont trois coauteurs français, l'illustre avec brio dans un article fascinant de Nature [1] montrant comment processus mécaniques et moléculaires s'intriquent lors des premiers évènements de différenciation dans l'embryon préimplantatoire de souris.

\section{Rupture de la symétrie chez l'embryon} de mammifère

On sait que chez les mammifères, les blastomères issus des premières divisions du zygote sont peu connectés, partagent un profil moléculaire très similaire et un potentiel équivalent (chaque blastomère totipotent peut se développer en un individu viable). Mais rapidement, à partir du stade 8 cellules (jour 2,5 chez la souris) correspondant à la compac- tion (processus d'adhérence très forte entre blastomères), cette symétrie est rompue: les blastomères se polarisent, et, vers le stade 16 cellules, leur position spatiale n'est plus équivalente: certains occupent une position périphérique, d'autres une position centrale [2]. Cette asymétrie aboutit à l'émergence de deux lignages: les blastomères en périphérie adoptent un destin de trophectoderme, qui formera le placenta, ceux du centre forment la «masse interne » à l'origine de l'embryon. Plusieurs modèles ont été proposés pour rendre compte de la rupture de cette symétrie et de l'émergence des deux destins cellulaires [2, 3]. Au stade 8 cellules, les blastomères sont polarisés: on distingue un domaine apical où s'exprime la aPKC (atypical protein kinase (). Selon le plan de division dans l'espace, ce domaine apical est hérité soit uniquement par la cellule fille « apicale » (division asymétrique), soit par les deux cellules filles (division symétrique). Dans le premier cas de figure, la cellule fille ayant hérité de ce domaine apical est polarisée (comme la cellule parentale) et s'engage vers un destin trophectoderme; la cellule fille dépourvue de domaine apical contribuera à la masse interne (Figure 1). Restait à étudier le mécanisme utilisé par les cellules pour adopter une position interne ou externe. Résulte-t-il seulement du positionnement basal d'une des cellules filles issues de la division asymétrique d'une cellule parentale dont le fuseau mitotique aurait une orientation apicobasale? Peut-on au contraire démontrer un processus de tri cellulaire sélectif?
Contractilité cellulaire et internalisation des cellules de la masse interne : un processus de tri sélectif fondé sur des paramètres physiques Une première approche a consisté à étudier la relation entre polarisation et contractilité, en mesurant la contractilité des blastomères. On sait que les embryons de souris à partir de 8 cellules se contractent périodiquement, ce qui contribue au processus de compaction [4]. Or, au stade 16 cellules, seuls les blastomères non polarisés sont doués de contractilité (présence de myosine), alors que les blastomères polarisés restent en périphérie et sont très peu contractiles (la aPKC du domaine apical s'oppose à la fonction de la myosine). Cette contractilité explique-t-elle le processus physique d'internalisation des blastomères destinés à former la masse interne?

Depuis quelques années, on sait que des paramètres mécaniques, régis par les lois physiques, participent de façon déterminante aux processus du développement embryonnaire, et sont couplés aux processus génétiques et moléculaires. La forme des cellules, leurs contacts, leur arrangement spatial, sont contrôlés par les tensions de surface entre les cellules, mais aussi entre les cellules et le milieu environnant [5]. Or, ces tensions de surface sont contrôlées par la contractilité cellulaire induite par le système actine-myosine. Afin d'analyser avec précision le processus d'internalisation, les auteurs de l'article ont imaginé un modèle théorique de doublet, qui permet d'analyser les forces impliquées; ce dernier repose sur trois paramètres: le degré de compaction, le rapport des tensions entre cellules voisines et entre cellules et milieu, et le 


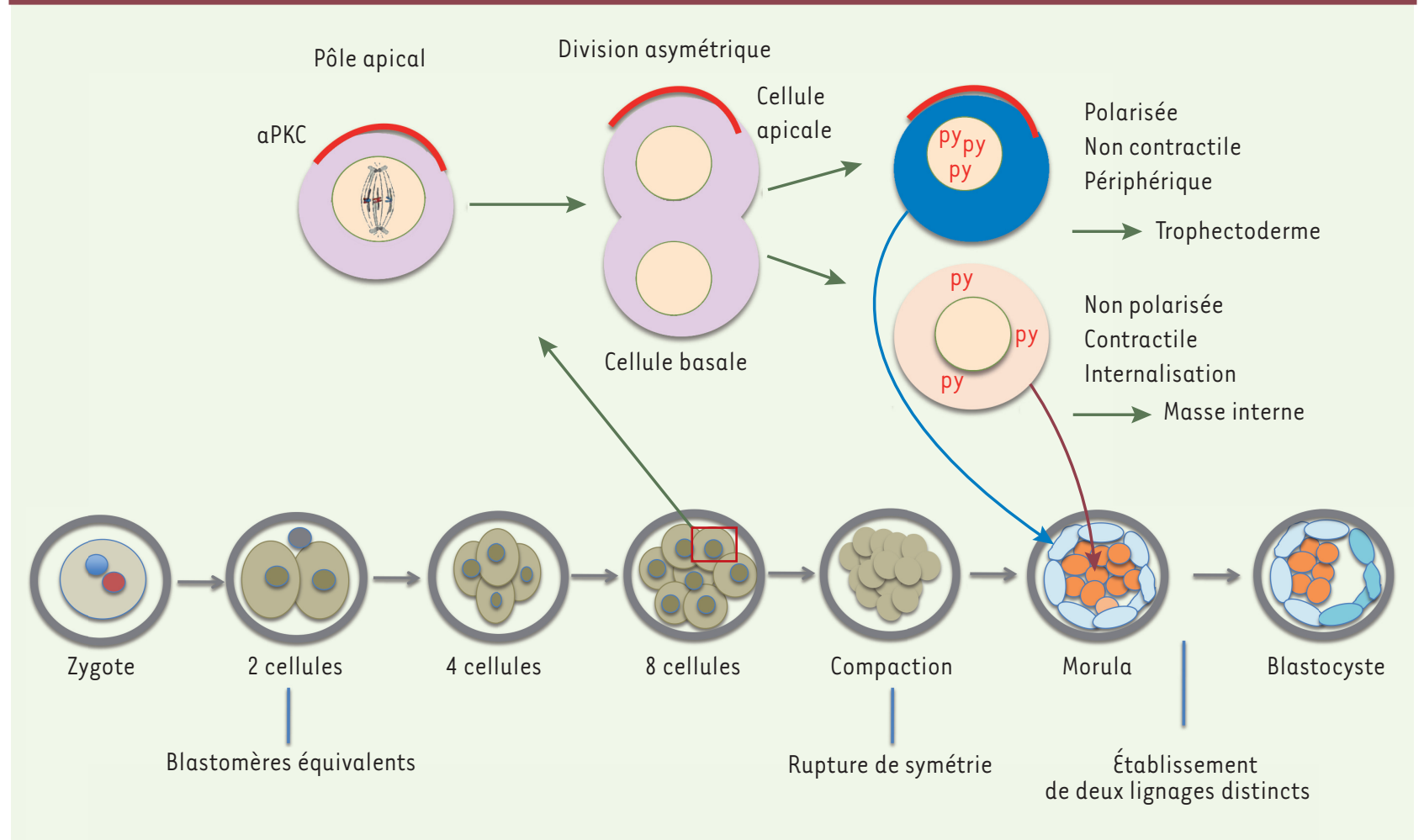

Figure 1. Rupture de symétrie lors de la division de blastomères de l'embryon de souris. Dans la partie inférieure du schéma, les premières divisions embryonnaires sont figurées, jusqu'au stade de blastocyste. Les deux lignages sont clairement individualisés au stade de morula (16 cellules et plus). Une division asymétrique est représentée dans la partie supérieure du schéma. Le fuseau mitotique est orienté selon l'axe apico-basal : la cellule fille apicale conserve le domaine apical contenant la aPKC (atypical protein kinase C); elle n'est pas contractile et contribue au trophectoderme, via l'expression nucléaire de p-Yap (py, Yap [yes-associated protein] phosphorylée). La cellule basale est dépourvue de domaine apical ; contractile, elle migre en position interne et contribuera à la masse interne (qui donnera l'embryon).

volume des cellules

$[6](\rightarrow)$.

Dans ce modèle de doublet, il est pos-

$(\rightarrow)$ Voir la Nouvelle de J.L. Maitre et al., $\mathrm{m} / \mathrm{s} \mathrm{n}^{\circ} 2$, février 2013 page 147

sible de déterminer une valeur seuil d'asymétrie des tensions de surface au-delà de laquelle il y a internalisation d'une cellule par l'autre (ce processus d'englobement d'une cellule par l'autre est appelé $\rightarrow$ Voir la Nouvelle de A.A. Mailleux et al., $\mathrm{m} / \mathrm{s} \mathrm{n}^{\circ} 3$, mars 2008 , page 246 entose [7]) $(\rightarrow)$.

Ce modèle peut être interprété en 3D, et mime alors l'embryon multicellulaire. En fait, les mêmes lois physiques qui gouvernent l'internalisation d'une cellule par une autre dans le modèle du doublet s'appliquent également à l'internalisation de cellules dans le modèle multicellulaire. Ce processus s'apparente donc à un processus de tri cellulaire (cell sorting).

\section{Du modèle théorique à la réalité} du vivant

Pour confronter les valeurs seuils des tensions cellulaires calculées dans les modèles théoriques à la réalité du vivant, les auteurs ont mesuré la tension de surface des deux cellules filles d'un blastomère après la division, et suivi leur positionnement, périphérique ou interne, dans l'embryon. Une asymétrie de tension était identifiée uniquement lorsque l'une des cellules filles (issue d'une division asymétrique, une cellule étant polarisée, l'autre non) était internalisée et contribuait à la masse interne. Si les deux cellules filles restaient en périphérie (division symé- trique, les deux cellules étant polarisées), aucune différence de tension n'était mesurée. Un second argument en faveur de la fidélité du modèle théorique est apporté par l'examen d'embryons de souris mMyh9, invalidées génétiquement pour l'allèle maternel du gène Myh9 (isoforme de la chaîne lourde de la myosine, requise lors du développement préimplantatoire). La compaction de ces embryons est retardée, mais ils se développent en des souriceaux viables. Toutefois, un blastomère mutant mMyh9 transféré dans un embryon sauvage restera en périphérie - tendant à envelopper les autres blastomères - et ne sera pas internalisé. À l'inverse, un blastomère normal placé à la surface d'un embryon mMyh9 est internalisé et contribue à 
la masse interne. L'internalisation met donc en jeu un processus actif de tri cellulaire.

\section{Contractilité et localisation}

\section{des gènes maîtres spécifiques}

\section{du destin cellulaire}

Si modifier les forces contractiles des cellules permet de changer la position des cellules dans l'embryon, on peut se demander s'il existe un lien entre ces forces et l'expression des gènes maîtres déterminant le destin cellulaire, en trophectoderme ou embryon. On sait que chez la souris, la spécification trophectodermique est sous le contrôle de Yap (yes-associated protein) [8]. Quand la voie est active, Yap-phosphorylée ( $p$-Yap) est exclue du noyau, empêchant la transcription de gènes cibles. C'est le profil observé dans les cellules de la masse interne, alors que, dans les blastomères périphériques, la quantité de $p$-Yap cytoplasmique est faible et $p-Y a p$ est essentiellement nucléaire, conduisant à l'induction de gènes spécifiques du trophectoderme (dont $c d x 2$ ). Les auteurs démontrent que, expérimentalement, l'absence de contractilité (mutant Myh9, ou exposition de l'embryon à un inhibiteur pharmacologique de l'activité ATPasique de la myosine II), qui inhibe la déformation des blastomères périphériques, retentit sur la localisation de Yap, et, in fine, sur le destin des cellules, qui, toutes, adoptent un phénotype de type masse interne, quelle que soit leur position dans l'embryon. La contractilité assure le couplage entre la position spatiale et le destin des cellules. J.L. Maître et ses collègues émettent l'hypothèse que la répartition dans la cellule des déterminants du destin cellulaire puisse être sensible aux contraintes mécaniques lors des mouvements cellulaires; ainsi, le changement de localisation de $p$-yap accompagne le mouvement des cellules, et joue peut-être un rôle dans l'acquisition de leur position finale.

\section{Conclusion}

Cet article démontre très élégamment l'intrication des trois paramètres contrôlant le premier processus de spécification cellulaire de l'embryon préimplantatoire, celui qui distingue les blastomères destinés à former le trophectoderme et ceux qui formeront les tissus embryonnaires. La polarité assure une répartition asymétrique des protéines apicales lors de la division, et oriente les cellules basales vers l'intérieur de l'embryon; la contractilité forte des cellules internes (non polarisées) et la contractilité faible des cellules périphériques consolident ce tri spatial des cellules, et la distribution asymétrique des déterminants moléculaires dirigeant le destin cellulaire, qui dépendrait également de contraintes mécaniques. Ces données permettent d'ébaucher un schéma d'auto-organisation de l'embryon préimplantatoire in vivo, qui illustre de façon éclatante que les lois de la physique sont essentielles pour comprendre le vivant. Ces données en rappellent d'autres récentes, notamment l'organisation ordonnée et spontanée in vitro des dérivés des trois feuillets germinaux issus de la différenciation des cellules souches pluripotentes (embryonnaires et iPS [induced pluripotent stem cells]) selon un patron qui rappelle celui de la gastrulation in vivo [9]. Jacques Prost, dans un Éditorial de 2011 [10] au titre évocateur «mais où sont passés les gènes?» $\rightarrow$, rappelait que «les cellules sont capables de tester leur envi-

$\rightarrow$ Voir l'éditorial de J. Prost, $m / s n^{\circ} 12$, décembre 2011, page 1043 ronnement mécanique. Inversement, l'expression génétique contrôle les propriétés cellulaires y compris les propriétés mécaniques ». Le travail de JL Maître et ses collaborateurs est une superbe illustration de ce lien entre «génétique, mécanique et dynamique », qui nous dévoile un petit peu de la complexité du développement embryonnaire précoce. $\diamond$

First commitment decision in the embryo

\section{REMERCIEMENTS}

Nous remercions Jean-Léon Maitre de sa relecture et de ses commentaires sur ce texte.

\section{LIENS D'INTÉRÊT}

L'auteur déclare n'avoir aucun lien d'intérêt concernant les données publiées dans cet article.

\section{RÉFÉRENCES}

1. Maître JL, Turlier H, Illukkumbura R, et al. Asymmetric division of contractile domains couples cell positioning and fate specification. Nature 2016; $536: 344-8$.

2. Wennekamp S, Mesecke S, Nédélec F, Hiiragi, T.A selforganization framework for symmetry breaking in the mammalian embryo. Nat Rev Mol Cell Biol 2013 ; 14 : 452-9.

3. Chazaud C, Yamanaka Y. Lineage specification in the mouse preimplantation embryo. Development 2016 ; 143 : 1063-74.

4. Maître JL, Niwayama R, Turlier H, et al. Pulsatile cellautonomous contractility drives compaction in the mouse embryo. Nat Cell Biol 2015 ; 17 : 849-55.

5. Heisenberg CP, Bellaïche $Y$. Forces in tissue morphogenesis and patterning. Cell 2013; 153 : 948-62.

6. Maître JL, Berthoumieux H, Krens SF, et al. La mécanique de l'adhésion cellulaire dans la gastrulation du poisson zèbre. Med Sci (Paris) 2013; 29 : 147-50.

7. Mailleux AA, Overholtzer M, Brugge JS. L'entose, mort cellulaire par cannibalisme entre cellules tumorales. Med Sci (Paris) 2008 ; 24 : 246-8.

8. Hirate $\mathrm{Y}$, Hirahara $S$, Inoue $\mathrm{K}$, et al. Polaritydependent distribution of angiomotin localizes Hippo signaling in preimplantation embryos. Curr Biol 2013 ; 23 : 1181-94.

9. Warmflash A, Sorre B, Etoc F, et al. A method to recapitulate early embryonic spatial patterning in human embryonic stem cells. Nat Methods $2014 ; 11$ : 847-54.
10. Prost J. Mais où sont passés les gènes ? Med Sci (Paris) $2011 ; 27: 1043-4$.

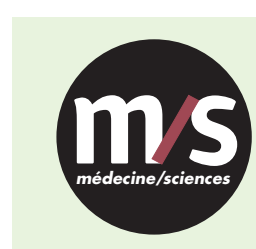

Tarifs d'abonnement $\mathrm{m} / \mathrm{s}-2017$

Abonnez-vous

à médecine/sciences
$>$ Grâce à $m / s$, vivez en direct les progrès des sciences biologiques et médicales

Bulletin d'abonnement page 202 dans ce numéro de $\mathrm{m} / \mathrm{s}$

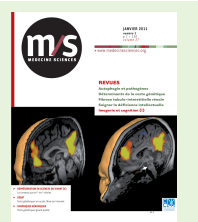

